\title{
Debye temperature and stiffness of carbon and boron nitride polymorphs from first principles calculations
}

$\operatorname{AUTHOR}(\mathrm{S})$ :

Tohei, T; Kuwabara, A; Oba, F; Tanaka, I

\section{CITATION:}

Tohei, T ... [et al]. Debye temperature and stiffness of carbon and boron nitride

polymorphs from first principles calculations. PHYSICAL REVIEW B 2006, 73(6): 064304.

ISSUE DATE:

2006-02

URL:

http://hdl.handle.net/2433/39879

RIGHT:

Copyright 2006 American Physical Society 
PHYSICAL REVIEW B 73, 064304 (2006)

\title{
Debye temperature and stiffness of carbon and boron nitride polymorphs from first principles calculations
}

\author{
Tetsuya Tohei,* Akihide Kuwabara, Fumiyasu Oba, and Isao Tanaka \\ Department of Materials Science and Engineering, Kyoto University, Yoshida, Sakyo, Kyoto 606-8501, Japan \\ (Received 14 September 2005; revised manuscript received 5 January 2006; published 23 February 2006)
}

\begin{abstract}
A theoretical investigation has been made on the phonon spectrum and heat capacity of polymorphs of carbon and boron nitride with special interests on the variation of Debye temperature and stiffness with temperature. A part of optical phonon branches of graphite exhibits higher frequencies than those of diamond. As a consequence, graphite shows smaller heat capacity and higher Debye temperature than diamond above a crossover temperature of $1000 \mathrm{~K}$. This supports experimental reports of heat capacity although available experimental data are widely scattered. The higher Debye stiffness of graphite at above $1000 \mathrm{~K}$ is not contradictory to the fact that conventional stiffness of diamond is much larger than that of graphite, since the Debye stiffness is determined by both acoustic and optical phonons, whereas only acoustic phonons contribute to the conventional stiffness. The same trend was found between hexagonal and cubic boron nitrides with a crossover temperature of $600 \mathrm{~K}$.
\end{abstract}

DOI: 10.1103/PhysRevB.73.064304

PACS number(s): 63.20.Dj, 65.40.-b, 81.05.Uw

\section{INTRODUCTION}

Allotropes of carbon show a striking contrast in their hardness. Diamond is the hardest natural substance we have ever known. Graphite, in contrast, is included in soft materials. The difference in these properties can be well explained in terms of chemical bondings. The crystal of diamond consists of three-dimensional networks of strong covalent bondings. On the other hand, the graphite crystal is made from stacking of two-dimensional hexagonal lattices with weak interlayer interaction which is the origin of the softness. Hardness usually means resistance to plastic deformation which can be measured by indentation tests. Stiffness is resistance to elastic deformation which can be measured by sound velocity in crystals. Acoustic phonons contribute to the sound propagation and therefore are related to the stiffness of crystals. There are $3 Z$ phonon branches in a crystal having a $Z$ atom unit cell. Only three of them are acoustic. The other 3Z-3 branches are called optical phonons. Although optical phonons do not contribute to the sound wave, they play important roles for heat capacity especially at elevated temperatures. In 1912 Debye proposed the theory of heat capacity, ${ }^{1}$ where the single Einstein frequency is replaced by a frequency spectrum. According to the Debye's model, temperature dependence of the heat capacity can be well defined using a single parameter called Debye temperature $\Theta_{D}$. It is related to the maximum phonon frequency $\nu_{D}$ of the frequency spectrum as $\Theta_{D}=h \nu_{D} / k_{B}$, where $h$ and $k_{B}$ are Planck's and Boltzmann's constant, respectively. Since vibrational frequency is proportional to square root of the stiffness within the harmonic approximation, both $\Theta_{D}$ and $\nu_{D}$ can be used as measures of the "stiffness" of solids. Hereafter we will call them Debye stiffness. It should be noted that the Debye stiffness defined in this way is different from the conventional stiffness that can be measured by acoustic waves as the resistance to acoustic phonon excitation. Yet, it is an important parameter to represent the resistance to overall phonon excitation including optical phonons. A widely accepted value of Debye temperature ${ }^{2}$ of diamond, $\Theta_{D}$ $=2240 \mathrm{~K}$, is much higher than that of graphite, $402 \mathrm{~K}$ when determined with low-temperature heat capacity data. Therefore, Debye stiffness is much higher in diamond at low temperatures. On the other hand, Wunderlich ${ }^{3}$ reported that the heat capacity of graphite becomes smaller than diamond at above $1000 \mathrm{~K}$, which was explained by the assumption that $\mathrm{C}-\mathrm{C}$ bonds in diamond is weaker than the conjugate double bonds in graphite. Similar experimental data have been reported by Atake. ${ }^{4}$ However, it is difficult to derive a definitive conclusion due to experimental uncertainties of the reported data. Here, let us quantitatively discuss the temperature dependence of the Debye stiffness using first principles calculations.

Theoretical phonon dispersions of diamond and graphite have been reported in several works. ${ }^{5-12}$ Results for boron nitride $(\mathrm{BN})$ polymorphs have also been reported. ${ }^{13-18}$ Very recently, Mounet and Marzari reported thermodynamical properties of carbon allotropes by first principles calculations. ${ }^{12}$ They succeeded in reproducing several experimental quantities including in-plane negative thermal expansion coefficient of graphite at low temperatures. To the authors' best knowledge, however, no systematic study has been made on the temperature dependence of the Debye stiffness of carbon allotropes or boron nitrides from first principles. Difference in the Debye stiffness between polymorphs has not been discussed, either.

\section{COMPUTATIONAL METHODS}

First principles total energy calculations were performed using VASP code. ${ }^{19}$ The interaction between ions and valence electrons was described by the projector augmented wave (PAW) method. ${ }^{20}$ The exchange and correlation effects were treated by the local density approximation (LDA). ${ }^{21}$ The $k$ mesh was sampled according to a Monkhorst-Pack scheme ${ }^{22}$ with a spacing of $0.4 / \AA$. The cutoff in the plane wave expansion was $500 \mathrm{eV}$. Convergence of relative energies with 
TABLE I. Calculated results of lattice constants, thermal expansion coefficients, and isothermal bulk moduli for four crystals. Experimental values are also shown for comparison (Refs. 30 and 32-37). Theoretical lattice constants are those obtained from calculations at 0 K. Volume thermal expansion and isothermal bulk modulus under finite temperatures were calculated within the quasiharmonic approximation.

\begin{tabular}{|c|c|c|c|c|c|c|c|c|}
\hline & \multicolumn{2}{|c|}{ Diamond } & \multicolumn{2}{|c|}{ Graphite } & \multicolumn{2}{|c|}{$c-\mathrm{BN}$} & \multicolumn{2}{|c|}{$h-\mathrm{BN}$} \\
\hline & calc & expt & calc & expt & calc & expt & calc & expt \\
\hline$a_{0}(\AA)$ & 3.536 & $3.567^{\mathrm{a}}$ & 2.447 & $2.460^{\mathrm{c}}$ & 3.582 & $3.615^{\mathrm{e}}$ & 2.490 & $2.504^{f}$ \\
\hline$c_{0}(\AA)$ & & & 6.660 & $6.700^{c}$ & & & 6.424 & $6.660^{f}$ \\
\hline$c_{0} / a_{0}$ & & & 2.722 & $2.724^{\mathrm{c}}$ & & & 2.580 & $2.660^{f}$ \\
\hline$V_{0}\left(\AA^{3} /\right.$ atom $)$ & 5.526 & $5.673^{\mathrm{a}}$ & 8.634 & $8.778^{c}$ & 5.745 & $5.905^{\mathrm{e}}$ & 8.623 & $9.041^{\mathrm{f}}$ \\
\hline$\beta 300 \mathrm{~K}\left(10^{-6} 1 / \mathrm{K}\right)$ & 3.35 & $3.75^{\mathrm{b}}$ & 19.8 & $21.9^{\mathrm{b}}$ & 5.33 & $5.4^{\mathrm{b}}$ & 29.8 & $30.1^{\mathrm{g}}$ \\
\hline$\beta 1000 \mathrm{~K}\left(10^{-6} 1 / \mathrm{K}\right)$ & 12.8 & $13.2^{\mathrm{b}}$ & 18.6 & $29.5^{\mathrm{b}}$ & 16.2 & $17.7^{\mathrm{b}}$ & 24.4 & \\
\hline$B_{0} 300 \mathrm{~K}(\mathrm{GPa})$ & 454 & $440-560^{\mathrm{a}}$ & 28.7 & $33.8^{\mathrm{d}}$ & 391 & $369-465^{a}$ & 26.3 & $37^{\mathrm{f}}$ \\
\hline
\end{tabular}

${ }^{\text {aReference } 30 .}$

${ }^{b}$ Reference 32 .

'Reference 33.

${ }^{\mathrm{d}}$ Reference 34 .

respect to the $k$ mesh and energy cutoff was found to be better than $1 \mathrm{meV} / \mathrm{atom}$.

Lattice dynamics has been computed by the direct method. ${ }^{23}$ Hellmann-Feynman forces exerted on all atoms in supercells by finite atomic displacements of every symmetrically nonequivalent atom were calculated. Supercells were composed of 64 atoms for diamond and $c$-BN and 128 atoms for graphite and $h$-BN. Displacements were $\pm 0.03 \AA$ for diamond and $c$-BN and $\pm 0.05 \AA$ for graphite and $h$-BN. ${ }^{24}$ Force constants matrix and then the dynamical matrix were constructed. Phonon frequencies were obtained by the diagonalization of the dynamical matrix. In polar crystals, a macroscopic electric field arising from ionic displacements leads to nonanalytical term of the dynamical matrix at wave vector $k=0$. This is observed as a splitting between the frequencies of the longitudinal optical (LO) and transverse optical (TO) phonons at $\Gamma$ point. ${ }^{25}$ In the present calculations, the nonanalytical behavior of the dynamical matrix has been treated by a conventional manner using the Born effective charge tensor and the electronic part of the dielectric constant. ${ }^{26}$ Born effective charges were calculated by the Berry phase approach. ${ }^{27,28}$ We obtained the value of $1.93(-1.93)$ for boron (nitrogen) in cubic $\mathrm{BN}$, and $2.70(-2.70)$ and 0.82 $(-0.82)$ for the direction parallel and perpendicular to the basal plane of hexagonal BN. They are in good agreement with previously reported values by linear response calculations. $^{29}$ Dielectric constants for infinite frequency limit were taken from the experimental values. ${ }^{30}$

To evaluate thermodynamical properties under finite temperatures, we have employed quasi harmonic approximation (QHA) ${ }^{31}$ Free energy of crystals at a given volume was obtained as a sum of the static total energy and the vibrational free energy. The vibrational contribution to the free energy was calculated as

$$
F_{\mathrm{vib}}=r N_{A} k_{B} T \int_{0}^{\infty} d \nu g(\nu) \ln \left[2 \sinh \left(h \nu / 2 k_{B} T\right)\right],
$$

where $r$ is the number of degree of freedom in the unit cell and $N_{A}$ is Avogadro's constant. Equilibrium volume and bulk
${ }^{\mathrm{e}}$ Reference 35 .

fReference 36.

${ }^{g}$ Reference 37.

modulus at a given temperature were obtained from the volume dependence of free energy by fitting the curve to the third order Birch-Murnaghan equation of state. Heat capacity at constant volume $C_{V}$ can be calculated directly from the phonon DOS under the harmonic approximation. Molar heat capacity was obtained by integrating the product of the DOS $g(\nu)$ and the weighting factor $W\left(h \nu / k_{B} T\right)$ $=\left(h \nu / k_{B} T\right)^{2} \exp \left(h \nu / k_{B} T\right) /\left[\exp \left(h \nu / k_{B} T\right)-1\right]^{2}$ as

$$
C_{V}=r N_{A} k_{B} \int_{0}^{\infty} d \nu g(\nu) W\left(h \nu / k_{B} T\right) .
$$

The difference between $C_{p}$ and $C_{V}$ was given by a thermodynamical relationship $C_{p}-C_{V}=V T \beta^{2} B_{0}$, where $V, T, \beta$, and $B_{0}$ represents molar volume, absolute temperature, volume thermal expansion coefficient, and isothermal bulk modulus, respectively.

Heat capacity at constant volume under the Debye approximation is expressed as follows:

$$
C_{V}=9 N_{A} k_{B}\left(T / \Theta_{D}\right)^{3} \int_{0}^{\Theta_{D} / T} d \nu\left(h \nu / k_{B} T\right)^{2} W\left(h \nu / k_{B} T\right) .
$$

Debye temperature at a given temperature was determined by fitting the theoretical $C_{V}$ to that from this formula.

\section{RESULTS AND DISCUSSION}

Structural and elastic parameters obtained by the calculations are summarized in Table I together with experimental values. Structural parameters are within usual errors of LDA for all crystals. Elastic properties also agree well with experimental values, except for a failure in predicting thermal expansion $\beta$ at $1000 \mathrm{~K}$ for graphite. Although the reason for the failure is not clearly ascertained, it may be attributed to the limitation of the LDA based calculations for graphitic structures with extended interlayer separations. Mounet and Marzari $^{12}$ adopted generalized gradient approximation (GGA) instead of LDA for a systematic calculation of the 

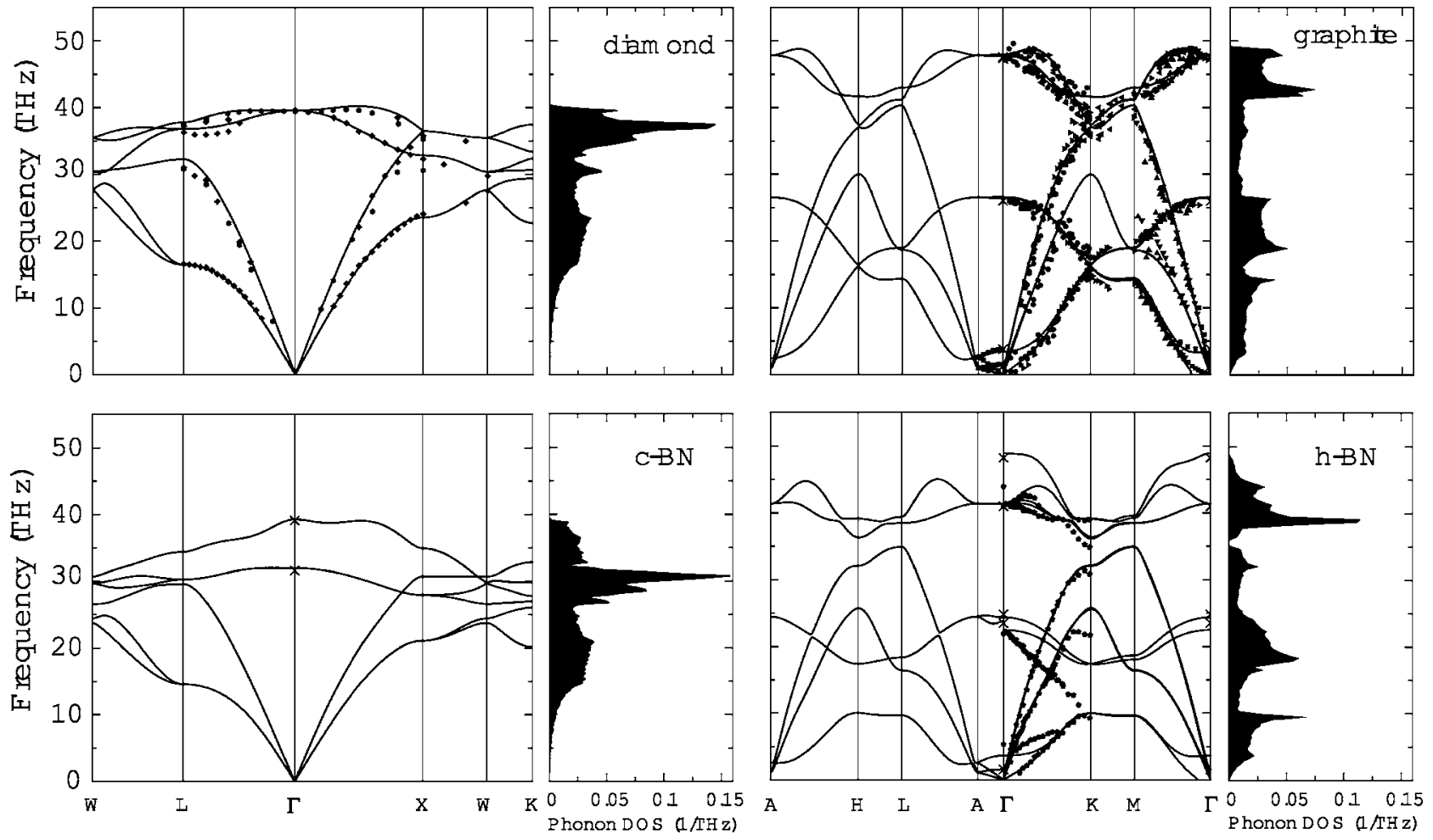

FIG. 1. Phonon dispersion relations and phonon density of states of four crystals. Solid curves show the calculated dispersions. Experimentally, dispersion relations over the Brillouin zone have been obtained by measurements such as neutron scattering, electron energy-loss spectroscopy, and x-ray scattering techniques. Experimental values from different data sources are discriminated by different symbols (Refs. 9 and 38-45). Frequencies at $\Gamma$ point have been determined by Raman and IR spectrum experiments, which are denoted by crosses (Refs. 46-50).

thermal expansion of graphite crystal within QHA. They failed to obtain the equilibrium interlayer separation of graphite. Although they employed experimental value of the ratio of lattice constants $c / a$ as a remedy for the GGA's failure, they could not reproduce the out-of plane thermal expansion coefficient above $150 \mathrm{~K}$. Although the error in $\beta$ in the present study is not negligible, we use the theoretical $\beta$ to evaluate $C_{p}$. The error in $C_{p}$ does not change the presence of the heat capacity cross-over that will be mentioned in detail later in this paper.

Figure 1 shows the calculated results for phonon dispersion relation and phonon density of states (DOS) for four crystals. Present results well trace the main features of theoretical phonon dispersion relations of carbon $^{5-12}$ and boron nitride ${ }^{13-18}$ in literature. Overall agreements of the calculated frequencies with the experimental values are quite good, including the low frequency modes in graphite where interactions between adjacent layers are essential. In diamond, the phonon DOS in low-frequency region rises up with $\nu^{2}$ dependency, which is typical for simple acoustic modes of three-dimensional lattices as described in the Debye model. The phonon spectrum has a prominent peak in the highest frequency region of near $37 \mathrm{THz}$, which consists of optical modes associated with bending of $s p^{3}$ bonds. The phonon spectrum of graphite, on the other hand, shows quite different features from that of diamond. First it has a band of vibrational states ranging from low- to middle-frequency regions. Such a low frequency band mainly consists of modes concerning layer-shearing, layer-breathing, and layerbending motions. In a high-frequency region, we can recognize another band of vibrational states, whose frequencies are even higher than the maximum frequency in diamond. Atomic displacements in this region correspond to in-plane bond-stretching modes. Similar observations are made in the comparison between two polymorphs of boron nitride.

Figures 2(a) and 2(b) illustrate the temperature variation of weighted phonon DOS $g(\nu) W\left(h \nu / k_{B} T\right)$, namely, the integrand in the formula of the heat capacity, for diamond and graphite in three-dimensional plots. The weighting factor represents how much proportion of the vibrational states can be excited and contributes to the lattice heat capacity. At $100 \mathrm{~K}$, for instance, $W\left(h \nu / k_{B} T\right)$ is smaller than $1 \%$ in the frequency region above $20 \mathrm{THz}$. Only low-frequency states can therefore contribute to the heat capacity at low temperatures. The lower panels of the figure [Figs. 2(c) and 2(d)] show cross sections of the three-dimensional plots at several temperatures. Heat capacity is proportional to the cross section at a given temperature by definition. It may be instructive to look at the three-dimensional plot with analogy to a "vase." Heat corresponds to "water" that is poured into the "vase." Larger amount of "water" is required to increase the "water surface" in the "vase" when the cross section is larger. Larger amount of heat is required to increase the temperature when the cross section is larger, which corresponds to the larger heat capacity.

Since graphite has much more vibrational states in a low frequency region than diamond does, graphite exhibits larger 

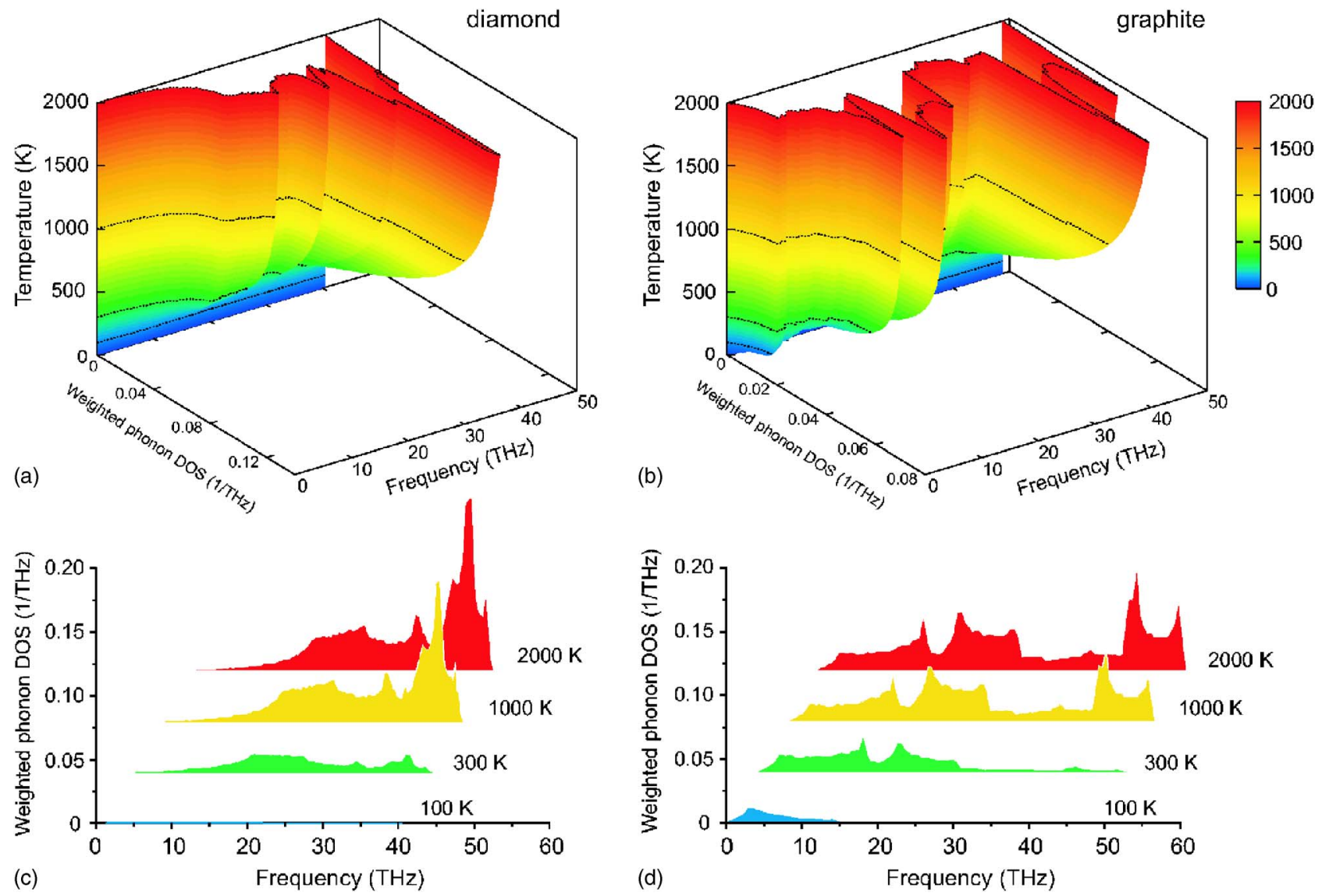

FIG. 2. (Color online) Temperature variation of weighted phonon DOS. (a) Weighted phonon DOS of diamond. (b) Weighted phonon DOS of graphite. The weighted phonon DOS, $g(\nu) W\left(h \nu / k_{B} T\right)$, is the integrand in the formula of heat capacity and represents the portion of phonon DOS that contributes to the heat capacity. Lower panels show cross sections of the weighted phonon DOS at several temperatures for (c) diamond, and (d) graphite. Curves for 300, 1000, $2000 \mathrm{~K}$ are offset for $x$ and $y$ directions by $4 \mathrm{THz}$ and $0.041 / \mathrm{THz}$, respectively. Heat capacity is proportional to the cross section at a given temperature.

I'm' I'm heat capacity than diamond at low temperatures. We can see that at an elevated temperature of $1000 \mathrm{~K}$, on the other hand, almost all vibrational states in the whole frequency range can be excited in both of crystals.

Calculated heat capacities for four crystals are shown in Fig. 3 together with experimental data. Heat capacity at constant pressure $C_{p}$, which is the usual condition for experiments, was calculated within the QHA. Lower panels of the figure [Figs. 3(c) and 3(d)] show differences in heat capacities between two crystals $\Delta C_{p}$ and $\Delta C_{V}$. The calculated values of $C_{p}$ show good agreement to experimental values, although experimental data are widely scattered especially at high temperatures and some of them deviate significantly. The scattering prevents us from discussing high temperature heat capacities only from experimental values. Information by reliable theoretical calculations is therefore very useful for detailed discussion. It is interesting that $\Delta C_{V}$ becomes negative at above $1000 \mathrm{~K}$ for graphite/diamond.

By fitting the theoretical $C_{V}$ with the Debye model, one can derive temperature dependent Debye temperature $\Theta_{D}(T)$. Results are presented in Fig. 4. Experimental Debye temperatures are typically determined by fitting the heat capacity at low temperatures. The agreement of the present $\Theta_{D}(T)$ with the experimental values at 0 and $298 \mathrm{~K}$ is quite satis- factory. In graphite, the temperature dependence of $\Theta_{D}(T)$ is found to be much more significant than that of diamond making a crossover at $1000 \mathrm{~K}$. This can be easily correlated to the shape of phonon DOS as shown in Fig. 1. Since $\Theta_{D}(T)$ corresponds to the Debye stiffness as discussed earlier in this report, we can reach the conclusion that the Debye stiffness is greater in diamond than in graphite only below $1000 \mathrm{~K}$.

The same trend can be seen in BN polymorphs. Hexagonal $\mathrm{BN}(h-\mathrm{BN})$ with $s p^{2}$ type bondings show higherfrequency phonon modes than cubic $\mathrm{BN}(c-\mathrm{BN})$ with $s p^{3}$ type bondings. Both $C_{V}$ and $\Theta_{D}(T)$ undergoes the crossover at $600 \mathrm{~K}$. Above that temperature, the Debye stiffness of $h$-BN is larger than that of $c$-BN. The lower crossover temperature in $\mathrm{BN}$ than $\mathrm{C}$ can be ascribed to the lower phonon frequency in $\mathrm{BN}$. The crossover behavior found in this work may be a general trend among polymorphs that are composed of different dimensionalities or types of chemical bondings.

\section{SUMMARY}

First principle PAW calculations within LDA were made on the phonon spectrum and heat capacity of diamond, in comparison to its allotrope, graphite. The same set of calcu- 

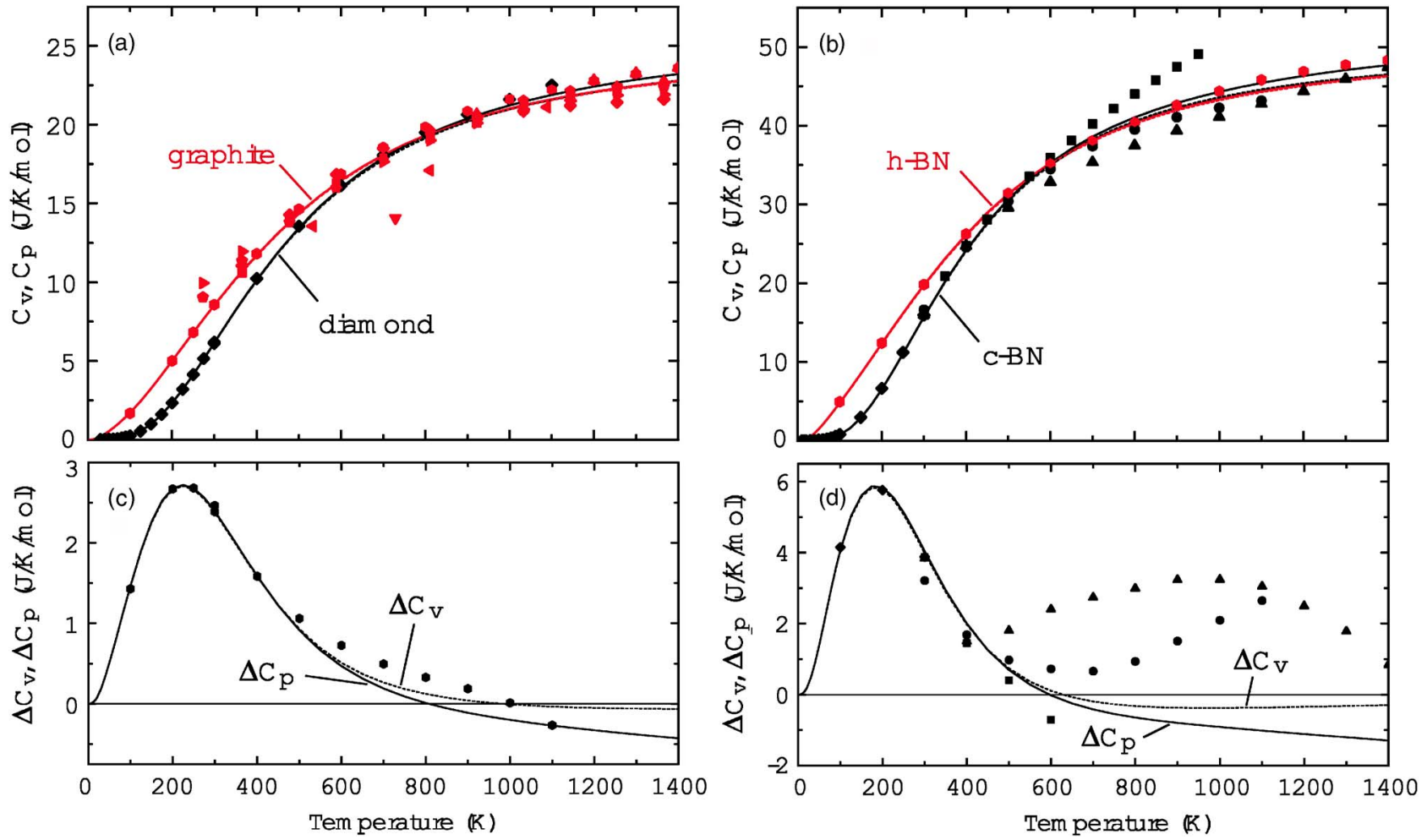

FIG. 3. (Color online) Heat capacity and difference in heat capacity of related polymorphs. (a) Heat capacity of carbon allotropes. (b) Heat capacity of boron nitride polymorphs. Solid and dashed curves represent the theoretical values of $C_{p}$ and $C_{V}$, respectively. Experimental values from different data sources are denoted by different symbols (Refs. 51-54). (c) Difference in heat capacity of two carbon allotropes calculated as, $\Delta C=C$ (graphite) $-C$ (diamond). (d) Heat capacity difference of boron nitride polymorphs given as $\Delta C=C(h$-BN) $-C(c$-BN). Experimental values of heat capacity differences were obtained from smoothed curves available in literature.

lations was made for hexagonal and cubic BN. Major results can be summarized as follows.

(1) Lattice constants, thermal expansion coefficients and isothermal bulk modulus agree well with experimental values for all of four crystals, except for a failure in predicting thermal expansion $\beta$ at $1000 \mathrm{~K}$ for graphite.

(2) Theoretical phonon dispersion relation and phonon density of states reproduce experimental data quite satisfactorily as in previous works in literature.
(3) A part of optical branches of graphite shows higher phonon frequencies than those of diamond. As a consequence, graphite shows smaller heat capacity and higher Debye temperature than diamond at temperatures above the crossover temperature of $1000 \mathrm{~K}$. This supports experimental reports of heat capacity although the experimental data are widely scattered.

(4) Consequently graphite shows higher Debye stiffness than diamond above $1000 \mathrm{~K}$, which may be contrary to the
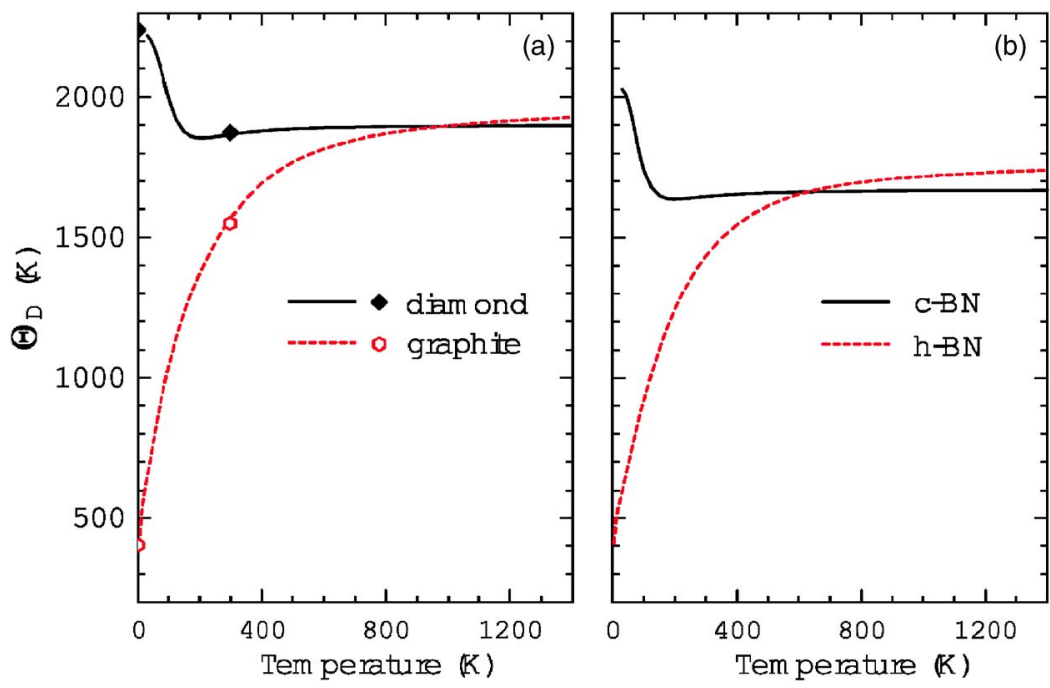

FIG. 4. (Color online) Temperature dependence of Debye temperature $\Theta_{D}$ for four crystals. (a) diamond and graphite; (b) cubic and hexagonal boron nitride. Theoretical and experimental values are shown by curves and symbols, respectively. Theoretical values of $\Theta_{D}$ are determined in such a way that the heat capacity by the Debye model [Eq. (3)] corresponds to the calculated values of $C_{V}$. Experimental values of $\Theta_{D}$ presented here were obtained from heat capacity measurements (Ref. 2). 
intuition that diamond is stiffer than graphite. This can be ascribed to the fact that both acoustic and optical phonons contribute to the Debye stiffness, whereas only acoustic phonons contribute to the conventional stiffness measured by the sound propagation.

(5) The same trend can be found for boron nitride polymorphs with the crossover temperature of $600 \mathrm{~K}$. The crossover behavior seems to be a general trend among polymorphs that are composed of different dimensionalities or types of chemical bondings.

\section{ACKNOWLEDGMENTS}

We acknowledge T. Suga, T. Yamamoto, and K. Tsujii for valuable comments on the study. This work was supported by projects from the Ministry of Education, Culture, Sports, Science and Technology (MEXT). They are the 21st century COE program and Grant-in-Aids for Young Scientists (B). T. T. also acknowledges support from Japan Society for the Promotion of Science (JSPS).
*Author to whom correspondence should be addressed. Email address: tohei@t02.mbox.media.kyoto-u.ac.jp

${ }^{1}$ P. Debye, Ann. Phys. 39, 789 (1912).

${ }^{2}$ K. A. Gschneidner, Jr., in Solid State Physics, edited by F. Seitz and D. Turnbull (Academic Press, New York, 1964), Vol. 16, pp. 275-426.

${ }^{3}$ B. Wunderlich and Y. Jin, Thermochim. Acta 226, 169 (1993).

${ }^{4}$ T. Atake, J. Crystallogr. Soc. Jpn. 44, 35 (2002) (in Japanese).

${ }^{5}$ P. Pavone, K. Karch, O. Schütt, W. Windl, D. Strauch, P. Giannozzi, and S. Baroni, Phys. Rev. B 48, 3156 (1993).

${ }^{6}$ G. Kresse, J. Furthmuller, and J. Hafner, Europhys. Lett. 32, 729 (1995).

${ }^{7}$ P. Pavone, R. Bauer, K. Karch, O. Schütt, S. Vent, W. Windl, D. Strauch, S. Baroni, and S. de Gironcoli, Physica B 219\&220, 439 (1996).

${ }^{8}$ O. Dubay and G. Kresse, Phys. Rev. B 67, 035401 (2003).

${ }^{9}$ J. Maultzsch, S. Reich, C. Thomsen, H. Requardt, and P. Ordejon, Phys. Rev. Lett. 92, 075501 (2004).

${ }^{10}$ L. Vitali, M. A. Schneider, K. Kern, L. Wirtz, and A. Rubio, Phys. Rev. B 69, 121414(R) (2004).

${ }^{11}$ L. Wirtz and A. Rubio, Solid State Commun. 131, 141 (2004).

${ }^{12}$ N. Mounet and N. Marzari, Phys. Rev. B 71, 205214 (2005).

${ }^{13}$ K. Karch, F. Bechstedt, P. Pavone, and D. Strauch, Physica B 219\&220, 445 (1996).

${ }^{14}$ G. Kern, G. Kresse, and J. Hafner, Phys. Rev. B 59, 8551 (1999).

${ }^{15}$ K. Parlinski, J. Alloys Compd. 328, 97 (2001).

${ }^{16}$ F. Bechstedt, U. Grossner, and J. Furthmuller, Phys. Rev. B 62, 8003 (2000).

${ }^{17}$ H. W. L. Alves, J. L. A. Alves, J. L. P. Castineira, and J. R. Leite, Mater. Sci. Eng., B 59, 264 (1999).

${ }^{18}$ W. J. Yu, W. M. Lau, S. P. Chan, Z. F. Liu, and Q. Q. Zheng, Phys. Rev. B 67, 014108 (2003).

${ }^{19}$ G. Kresse and J. Furthmüller, Phys. Rev. B 54, 11169 (1996).

${ }^{20}$ P. E. Blöchl, Phys. Rev. B 50, 17953 (1994).

${ }^{21}$ J. P. Perdew and A. Zunger, Phys. Rev. B 23, 5048 (1981).

${ }^{22}$ H. J. Monkhorst and J. D. Pack, Phys. Rev. B 13, 5188 (1976).

${ }^{23}$ K. Parlinski, Z.-Q. Li, and Y. Kawazoe, Phys. Rev. Lett. 78, 4063 (1997).

${ }^{24}$ We have checked in diamond that changing the amplitude of the displacements between 0.01 and 0.05 angstrom causes no noticeable change in the frequency and the heat capacity: maximum changes in the Gamma point frequency and the $C_{V}$ at $1000 \mathrm{~K}$ were found to be about 0.1 and $0.04 \%$.

${ }^{25}$ LO-TO splitting affects only infrared active LO modes near Gamma point, raising the frequencies of LO modes above those of TO modes. Since the region is rather small and thermodynamics samples the whole Brillouin zone, we can expect the influence of the LO-TO splitting on thermodynamic functions to be small. We found that neglecting LO-TO splitting in $c$-BN results in only tiny changes of the $C_{V}$ and the Debye temperature at $1000 \mathrm{~K}: 0.04 \mathrm{~J} / \mathrm{K} / \mathrm{mol}$ and $5 \mathrm{~K}$, respectively.

${ }^{26}$ K. Parlinski, Z. Q. Li, and Y. Kawazoe, Phys. Rev. Lett. 81, 3298 (1998).

${ }^{27}$ R. D. King-Smith and D. Vanderbilt, Phys. Rev. B 47, R1651 (1993).

${ }^{28}$ R. Resta, Rev. Mod. Phys. 66, 899 (1994).

${ }^{29}$ N. Ohba, K. Miwa, N. Nagasako, and A. Fukumoto, Phys. Rev. B 63, 115207 (2001).

${ }^{30}$ Group IV Elements, IV-IV and III-V Compounds, edited by U. Rössler, Vol. 41A1 of Landolt Börnstein, Group III Condensed Matter (Springer-Verlag, Berlin, 2001).

${ }^{31}$ A. A. Maradudin, E. W. Montroll, G. H. Weiss, and I. P. Ipatova, Theory of Lattice Dynamics in the Harmonic Approximation, 2nd ed. (Academic, New York, 1971).

${ }^{32}$ Y. S. Touloukian, R. K. Kirby, R. E. Taylor, and T. Y. R. Lee, Thermal Expansion: Nonmetallic Solids, Vol. 13 of Thermophysical Properties of Matter (IFI-Plenum, New York, 1977).

${ }^{33}$ Y. Baskin and L. Meyer, Phys. Rev. 100, 544 (1955).

${ }^{34}$ M. Hanfland, H. Beister, and K. Syassen, Phys. Rev. B 39, 12598 (1989).

${ }^{35}$ R. H. Wentorf, Jr., J. Chem. Phys. 26, 956 (1957).

${ }^{36}$ V. L. Solozhenko, G. Will, and F. Elf, Solid State Commun. 96, 1 (1995).

${ }^{37}$ B. Yates, M. J. Overy, and O. Pirgon, Philos. Mag. 32, 847 (1975).

${ }^{38}$ J. L. Warren, J. L. Yarnell, G. Dolling, and R. A. Cowley, Phys. Rev. 158, 805 (1967).

${ }^{39}$ J. L. Wilkes, R. E. Palmer, and R. F. Willis, J. Electron Spectrosc. Relat. Phenom. 44, 355 (1987).

${ }^{40}$ S. Siebentritt, R. Pues, K. H. Rieder, and A. M. Shikin, Phys. Rev. B 55, 7927 (1997).

${ }^{41}$ R. Nicklow, N. Wakabayashi, and H. G. Smith, Phys. Rev. B 5, 4951 (1972).

${ }^{42}$ C. Oshima, T. Aizawa, R. Souda, and Y. Ishizawa, Solid State Commun. 65, 1601 (1988).

${ }^{43}$ M. Schwoerer-Bohning, A. T. Macrander, and D. O. Arms, Phys. Rev. Lett. 80, 5572 (1998).

${ }^{44}$ H. Yanagisawa, T. Tanaka, Y. Ishida, M. Matsue, E. Rokuta, S. Otani, and C. Oshima, Surf. Interface Anal. 37, 133 (2005).

${ }^{45}$ E. Rokuta, Y. Hasegawa, K. Suzuki, Y. Gamou, C. Oshima, and 
A. Nagashima, Phys. Rev. Lett. 79, 4609 (1997).

${ }^{46}$ R. Al-Jishi and G. Dresselhaus, Phys. Rev. B 26, 4514 (1982).

${ }^{47}$ J. A. Sanjurjo, E. Lopez-Cruz, P. Vogl, and M. Cardona, Phys. Rev. B 28, 4579 (1983).

${ }^{48}$ T. Werninghaus, J. Hahn, F. Richter, and D. R. T. Zahn, Appl. Phys. Lett. 70, 958 (1997).

${ }^{49}$ R. Geick, C. H. Perry, and G. Rupprecht, Phys. Rev. 146, 543 (1966).

${ }^{50}$ R. J. Nemanich, S. A. Solin, and R. M. Martin, Phys. Rev. B 23, 6348 (1981).
${ }^{51}$ Y. S. Touloukian and E. H. Buyco, Specific Heat: Nonmetallic Solids, Vol. 5 of Thermophysical Properties of Matter (IFIPrenum, New York, 1970).

${ }^{52} \mathrm{M}$. W. Chase, Jr., NIST-JANAF Thermochemical Tables, 4th ed. [J. Phys. Chem. Ref. Data 9 1998].

${ }^{53}$ V. L. Solozhenko, V. E. Yachmenev, V. A. Vilkovskii, A. N. Sokolov, and A. A. Shulzhenko, Russ. J. Phys. Chem. 61, 1480 (1987).

${ }^{54}$ V. E. Lyusternik and V. L. Solozhenko, Russ. J. Phys. Chem. 66, 629 (1992). 